\title{
Douleur et qualité de vie liée à la santé chez les personnes souffrant d'ulcères chroniques aux jambes
}

\author{
W. M. Hopman, MA (1, 2); M. Buchanan, PHCNP, M. Sc. (3); E. G. VanDenKerkhof, IA, Ph. D. - Santé publique (4, 5); \\ M. B. Harrison, IA, Ph. D. (4)
}

Cet article a fait l'objet d'une évaluation par les pairs.

\section{Résumé}

Introduction : L'ulcération veineuse de la jambe est associée à de la douleur et à une mauvaise qualité de vie liée à la santé (QVLS). Cette étude visait à définir les caractéristiques démographiques et cliniques associées à la douleur et à une diminution de la QVLS chez les patients présentant des ulcères veineux actifs.

Méthodologie : Les données de base obtenues dans le cadre de deux essais menés entre 2001 et 2007 (n = 564) ont été combinées. La douleur a été mesurée à l'aide de l'échelle numérique de la douleur (END), et la QVLS a été mesurée à l'aide du formulaire abrégé comportant 12 questions de l'Enquête sur la santé (SF-12), qui produit un sommaire de la composante physique (SCP) et un sommaire de la composante mentale (SCM). Les analyses ont notamment été effectuées par régression logistique (pour la douleur) et par régression linéaire (pour la QVLS).

Résultats : L’âge moyen était de 66,5 ans; 47 \% étaient des hommes. Le score médian sur l'END était de 2,2 (sur 10) et les scores moyens du SCP et du SCM étaient respectivement de 38,0 et de 50,5 (les scores sont normalisés à une moyenne de 50, qui représente la QVLS moyenne). Le jeune âge, le fait de vivre avec d'autres personnes et l'arthrite ont été associés à la douleur. Un score plus faible pour le SCP a été associé au fait d'être une femme, aux ulcères d'origine veineuse/mixte, aux gros ulcères, aux ulcères de longue durée, aux maladies cardiovasculaires, à l'arthrite et la douleur intense. Un score plus faible pour le SCM a été associé au jeune âge, aux ulcères de longue durée, aux affections concomitantes et à la douleur intense.

Conclusion : Il convient de mener des recherches pour mettre à l'essai des stratégies d'atténuation de la douleur et d'amélioration potentielle de la QVLS chez les groupes à risque élevé.

Mots-clés : chronique, ulcères aux jambes, SF-12, douleur, QVLS, formulaire abrégé à12 questions de l'Enquête sur la santé, qualité de vie liée à la santé

\section{Introduction}

Un ulcère veineux est une plaie chronique récurrente au niveau de la partie inférieure de la jambe. L'ulcération veineuse survient le plus souvent chez les adultes âgés, mais elle peut apparaître dans la vingtaine chez certains patients ${ }^{1,2}$. La prévalence des ulcères veineux actifs est estimée à 0,8 à 1 pour 1000 habitants $^{3}$. Les améliorations apportées à la pratique et l'organisation des soins ont grandement amélioré les taux de guérison ${ }^{4,5}$. Cependant, même après 12 mois de soins, $20 \%$ des ulcères demeurent non guéris $^{6}$ et jusqu'à $34 \%$ réapparaissent ${ }^{7,8}$.

Le fardeau de la maladie pour la population touchée est considérable. Les ulcères veineux ont un effet négatif sur la mobilité, l'emploi, les rôles sociaux, l'activité physique et la qualité de vie vi-12 $^{\text {La }}$ douleur est l'un des effets secondaires les plus souvent déclarés ${ }^{13}$ et, selon une étude de synthèse menée récemment, la douleur est le facteur le plus souvent désigné comme ayant une incidence sur la qualité de vie liée à la santé (QVLS) ${ }^{14}$. La guérison des ulcères et la QVLS sont au cœur des préoccupations des personnes atteintes et des fournisseurs de soins des plaies, car ces résultats témoignent de l'importance de la chronicité, de la récurrence et du fardeau des symptômes.

Selon la conceptualisation de Wilson et Cleary $^{15}$ à propos de la chronicité et du bien-être, la QVLS est constituée de quatre résultats en santé liés les uns aux autres dans une relation de cause à effet, et elle est influencée par des facteurs individuels et environnementaux. Les résultats en santé sont les facteurs biologiques, qui affectent l'état des symptômes, les deux pris ensemble affectant l'état fonctionnel. Le quatrième résultat en santé est la perception de la santé en général, qui est affectée à la fois par les trois précédents dans la relation de cause à effet et par les facteurs individuels et environnementaux.

Rattachement des auteurs :

1. Centre de recherche clinique, Hôpital général de Kingston, Kingston (Ontario), Canada

2. Département de santé communautaire et d'épidémiologie, Université Queen's, Kingston (Ontario), Canada

3. Centre de santé communautaire de Napanee et de la région, Napanee (Ontario), Canada

4. École des soins infirmiers, Université Queen's, Kingston (Ontario), Canada

5. Département d'anesthésiologie et de médecine préopératoire, Université Queen's, Kingston (Ontario), Canada

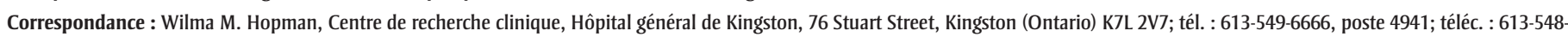
2428; courriel : hopmanw@kgh.kari.net 
Les facteurs biologiques concernent le fonctionnement des cellules, des organes ou de l'organisme et sont souvent choisis comme indicateurs de la gravité d'une maladie. Dans le contexte des ulcères veineux aux jambes, les facteurs biologiques incluent la taille, la durée et les causes des ulcères, de même que toute affection concomitante dont peut souffrir le patient. Pour ce qui est du lien entre la taille ou la durée des ulcères et la QVLS, les éléments de preuve ne concordent pas toujours $^{13,16-18}$. Les affections concomitantes chez les personnes souffrant de maladies veineuses n'ont pas été étudiées de manière approfondie, même si, en général, on considère que le diabète, l'insuffisance artérielle et les maladies auto-immunes retardent la guérison. Une étude a fait état d'un lien entre l'arthrose et la douleur ulcéreuse ${ }^{19}$.

L'état des symptômes est constituté de la perception qu'a une personne d'un état physique, psychologique ou cognitif anormal. L'ulcération est associée à de nombreux symptômes, dont la douleur, l'exsudat de la plaie, la démangeaison, l'enflure des membres inférieurs et les odeurs $^{20}$. De tous ces symptômes, la douleur est celui qui a fait l'objet du plus grand nombre d'études, peut-être parce qu'il existe beaucoup de méthodes éprouvées d'évaluation de la douleur ${ }^{14}$. La douleur peut avoir d'énormes conséquences et peut même pousser des personnes jeunes, autrement en bonne santé physique, à s'absenter, à prendre des jours de maladie et même à prendre une retraite anticipée ${ }^{21,22}$.

En 1995, Wilson et Cleary $^{15}$ ont défini l'état fonctionnel comme étant la capacité d'accomplir des tâches normales dans au moins quatre dimensions, soit les dimensions physique, sociale, relationnelle et psychologique. Les symptômes des maladies veineuses ont un effet négatif sur les activités quotidiennes ${ }^{11,20}$ et sur la fonction physique ${ }^{10}$. La mobilité peut être réduite par l'enflure ${ }^{21}$, et le fardeau des symptômes peut également influer négativement la santé mentale ${ }^{21,23}$. La perception de la santé en général, qui est une évaluation subjective globale de l'état de santé par le patient, représente l'intégration ou la synthèse des facteurs biologi- ques, de l'état des symptômes et de l'état fonctionnel ${ }^{15}$. Chez les personnes souffrant d'ulcération veineuse, le score de perception de la santé en général (mesurée à l'aide du formulaire comportant 36 questions de l'Enquête sur la santé, sur une échelle de 0 à 100) était inférieur de 3,3 à 13,3 points à ceux enregistrés chez la population de référence ${ }^{24-26}$.

Certains facteurs individuels peuvent également influencer l'état des symptômes, l'état fonctionnel, la perception de la santé et la qualité de vie. Pour ce qui est de l'incidence sur la QVLS, Price et Harding ${ }^{24}$ n'ont pas observé de différence entre les hommes et les femmes, mais Lindholm ${ }^{27}$ et Franks et collab. ${ }^{28}$ ont observé chez les hommes un effet plus important dans davantage de dimensions de la QVLS. Une autre étude a révélé un lien entre le fait de vivre avec un conjoint et l'amélioration de la QVLS ${ }^{29}$.

Notre étude avait comme objectif d'examiner le lien entre les variables cliniques, la douleur et la QVLS chez des patients présentant des ulcères veineux actifs. Nous nous sommes fondés sur le modèle de Wilson et Cleary $^{15}$ pour définir les variables d'intérêt pour les deux principales questions posées dans le cadre de notre étude : (1) l'âge, le sexe, les conditions de vie, les affections concomitantes ainsi que la taille et la durée des ulcères ont-ils une incidence sur l'intensité de la douleur? (2) Ces mêmes variables ainsi que l'intensité de la douleur ont-elles une incidence sur la QVLS physique et mentale?

\section{Méthodologie}

Notre échantillon était composé de personnes souffrant d'ulcères aux jambes et ayant participé à deux essais contrôlés randomisés (ECR) menés entre 2001 et $2007^{5,30}$. La guérison des ulcères était le principal résultat mesuré dans le cadre des deux essais, la QVLS et la douleur, les résultats secondaires. L'ensemble de données utilisé pour cette étude est constitué des données de l'évaluation transversale de base menée dans le cadre de ces deux essais. Les essais initiaux ont été jugés conformes aux règles d'éthique par le Comité d'éthique de la recherche de
l'Université Queen's et de ses hôpitaux universitaires affiliés (essai intitulé Canadian Bandaging ${ }^{30}$ ) et le Comité d'éthique de l'Institut de recherche en santé d'Ottawa (essai contrôlé randomisé intitulé Nurse Clinic Versus Home Delivery ${ }^{5}$ ); cette analyse combinée a été approuvée par le Comité d'éthique de la recherche de l'Université Queen's et de ses hôpitaux universitaires affiliés.

Tous les participants ont fait l'objet d'une évaluation complète, fondée sur des données probantes et effectuée par du personnel infirmier dans des installations de soins à domicile situées dans plusieurs collectivités canadiennes ainsi que dans des régions éloignées/rurales. Les participants aux deux études devaient être âgés de 18 ans ou plus, consentir à participer, ne pas être diabétiques et présenter un ulcère veineux ou un ulcère mixte artériel et veineux. Parmi les autres critères d'admissibilité figuraient la nécessité de présenter, pendant au moins une semaine, une plaie ouverte de la peau d'au moins $0,7 \mathrm{~cm}$ de largeur ou de hauteur sous le genou, ainsi qu'un indice de pression tibio-brachiale d'au moins 0,8 . Il pouvait s'agir d'un premier ulcère ou d'un ulcère récidivant.

La QVLS a été mesurée à l'aide du formulaire abrégé à 12 questions de l'Enquête sur la santé (SF-12) ${ }^{31}$. Le SF-12 permet d'obtenir deux scores, le sommaire de la composante physique (SCP) et le sommaire de la composante mentale (SCM). Le SCP, basé sur le formulaire original comportant 36 questions de l'Enquête sur la santé (SF-36), repose principalement sur les dimensions liées au fonctionnement physique, principalement sur les dimensions liées à la vitalité, au fonctionnement social, à la limitation des rôles due aux problèmes physiques, aux douleurs corporelles et à la perception de la santé en général, tandis que le SCM repose aux problèmes émotionnels et à la santé mentale ${ }^{32}$. Bien que les dimensions originales soient mesurées sur une échelle de 0 à 100, le SCP et le SCM sont normalisés à une moyenne de 50, les scores supérieurs et inférieurs à 50 étant respectivement considérés comme supérieurs et inférieurs à la moyenne ${ }^{31,32}$. Une différence de 2 à 3 points est considérée 
comme significative sur le plan clinique ${ }^{31}$. La fonction discriminante de l'outil s'est avérée efficace lors d'études sur de nombreux problèmes de santés3 et de deux études menées auprès d'échantillons de patients souffrant d'ulcères veineux aux jambes et fondées sur l'âge, la durée des ulcères et la mobilité ${ }^{17}$ ainsi que l'état de guérison à 12 mois $^{16}$. De nombreuses études sur les ulcères aux jambes ont utilisé cet outil pour mesurer la QVLS ou

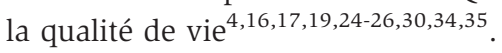

La douleur a été mesurée à l'aide de deux échelles : l'échelle numérique de la douleur (END) et l'indice de la douleur actuelle (IDA). L'END va de 0 (« aucune douleur ») à 10 (" douleur extrême »). Le score médian sur l'END a été utilisé pour établir la différence entre un groupe souffrant d'une douleur légère $(\leq 2,2)$ $10)$ et un groupe souffrant d'une douleur intense ( $>2,2 / 10)$. L'IDA consiste en six combinaisons de chiffres et de mots allant de 0 (" aucune douleur ») à 5 (" douleur atroce »). L'IDA fait partie de la version abrégée du Questionnaire McGill sur la douleur $^{36}$, et l'END et l'IDA ont tous deux été utilisés auprès de diverses populations cliniques, dont certaines souffrant d'ulcères veineux ${ }^{16,19,37-39}$.

Les variables biologiques comprenaient la taille, la durée et les causes des ulcères, ainsi que les affections concomitantes. La zone ulcéreuse a été mesurée par planimétrie informatisée et répartie en deux classes $\left(5 \mathrm{~cm}^{2}\right.$ ou moins; plus de $5 \mathrm{~cm}^{2}$ ). La mesure de la durée de l'ulcère était fondée sur la déclaration du patient et a été elle aussi répartie en deux classes (6 mois ou moins; plus de 6 mois). Les données relatives aux causes des ulcères et aux affections concomitantes ont été recueillies au moyen du Leg Ulcer Assessment Tool [outil d'évaluation des ulcères de la jambe ${ }^{40}$. Les patients ont indiqué leurs affections concomitantes pertinentes à partir d'une liste de différentes causes pouvant être à l'origine d'un ulcère, par exemple maladie cardiovasculaire, hypertension, maladie rénale, maladie artérielle des membres inférieurs et maladie intestinale inflammatoire. Les caractéristiques individuelles étaient définies comme suit : âge (moins de 65 ans; 65 ans et plus), sexe et soutien social (vivant seul; vivant avec d'autres personnes).

À l'aide de tests du khi carré, nous avons évalué le lien entre les variables nominales et les deux niveaux de mesure de la douleur, et nous avons utilisé des tests $t$ de Student pour évaluer le lien entre ces variables et les deux mesures continues de la QVLS. Nous avons ensuite utilisé une régression logistique (pour la douleur) et une régression linéaire (pour la QVLS) multivariée pour déterminer le sousensemble de principaux facteurs associés aux trois résultats. Le sexe a été forcé dans les modèles de régression à la lumière des données probantes préliminaires selon lesquelles les scores relatifs à la QVLS n'étaient pas identiques pour les hommes et les femmes souffrant d'ulcères aux jambes. Tous les modèles de régression utilisaient la saisie simultanée. Les variables étaient éliminées une à la fois en régressions successives si $p$ était supérieur à 0,10 et conservées si $p$ était inférieur ou égal à 0,10 , de façon à ne pas omettre de tendances importantes sur le plan clinique. Les rapports de cotes (RC) et les intervalles de confiance (IC) à $95 \%$ sont présentés pour les modèles multivariés finaux. Les analyses ont été effectuées à l'aide de la version 19.0 de SPSS pour Windows (IBM, Chicago, Illinois, ÉtatsUnis).

\section{Résultats}

Le profil sociodémographique et clinique des 564 participants à l'étude est assez typique de la population souffrant d'ulcères aux jambes (tableau 1). L'âge variait de 23 à 95 ans, avec une moyenne de 66,5 ans et un écart-type (É.-T.) de 15,$9 ; 340$ participants $(60,3 \%)$ avaient 65 ans ou plus. Il y avait plus d'hommes dans le groupe des moins de 65 ans $(127 / 224$, $57 \%$ ) et plus de femmes dans le groupe des 65 ans et plus $(201 / 340,59 \%)$, mais la répartition générale selon le sexe était similaire. Les ulcères étaient principalement causés par une maladie veineuse (488/564, 86,5 \%) touchant une jambe. La taille médiane des ulcères était de $3,4 \mathrm{~cm}^{2}$ et la durée médiane était de 2,6 mois. Environ $60 \%$ des sujets présentaient au moins une affection concomitante, l'arthrite étant la plus courante (211/564,
TABLEAU 1

Caractéristiques de l'échantillon de patients souffrant d'ulcères chroniques aux jambes $(n=564)$

\begin{tabular}{|c|c|}
\hline \multicolumn{2}{|l|}{ Caractéristiques des patients } \\
\hline Homme, n (\%) & $266(47,2)$ \\
\hline \multicolumn{2}{|l|}{ Âge, années } \\
\hline Moyen (É.-T.) & $66,5(15,9)$ \\
\hline Médian & 69,0 \\
\hline Plage & 23 à 95 \\
\hline 65 ans et plus, $n(\%)$ & $340(60,3)$ \\
\hline Vivant seul, n (\%) & $203(36,0)$ \\
\hline Fumeur actuel, n (\%) & $75(13,3)$ \\
\hline $\begin{array}{l}\text { Présence d'affections concomi- } \\
\text { tantes }^{\mathrm{a}}, \mathrm{n}(\%)\end{array}$ & $346(61,3)$ \\
\hline Arthrite & $211(37,4)$ \\
\hline Maladie cardiovasculaire ${ }^{\mathrm{b}}$ & $134(23,8)$ \\
\hline Hypertension & $183(32,4)$ \\
\hline Maladie rénale & $28(5,0)$ \\
\hline \multicolumn{2}{|l|}{ Cause probable, n (\%) } \\
\hline Veineuse & $488(86,5)$ \\
\hline Veineuse-mixte & $76(13,5)$ \\
\hline Ulcères bilatéraux, n (\%) & $21(3,7)$ \\
\hline \multicolumn{2}{|l|}{ Taille de l'ulcère } \\
\hline Moyenne & 9,98 \\
\hline Médiane & 3,41 \\
\hline Plage & 1 à 270 \\
\hline Plus de $5,0 \mathrm{~cm}^{2}, \mathrm{n}(\%)$ & $221(39,2)$ \\
\hline \multicolumn{2}{|l|}{ Durée de l'ulcère, mois } \\
\hline Moyenne & 8,7 \\
\hline Médiane & 2,6 \\
\hline Plage & 0,03 à 243,8 \\
\hline Plus de 6 mois, n (\%) & $292(51,8)$ \\
\hline
\end{tabular}

Abréviation : É.-T., écart-type.

${ }^{\text {a }}$ Vascularite, maladie intestinale inflammatoire, arthrite, maladie cardiovasculaire, hypertension, diabète, maladie rénale.

b Accident vasculaire cérébral, accident ischémique transitoire, maladie artérielle des membres inférieurs, infarctus du myocarde, angine, insuffisance cardiaque congestive.

$37,4 \%)$. Le cinquième des participants (114/564; 20,2\%) ont déclaré ne ressentir aucune douleur et 17,6\% (99/564) ont indiqué que leur douleur était " pénible» « terrible» ou « atroce» (tableau 2). Le score moyen pour le SCP était de 38,0, donc bien inférieur au score moyen de 50,5 de la population de référence au Canada, alors que le score moyen pour le SCM était de 50,5, donc similaire à celui de la population de référence $(51,7)^{41}$. 
TABLEAU 2

Caractéristiques de l'intensité de la douleur et de la qualité de vie liée à la santé des patients souffrant d'ulcères chroniques aux jambes $(n=564)$

\begin{tabular}{lc}
\multicolumn{2}{l}{ Résultats } \\
\hline END $^{\mathrm{a}}$ \\
Score moyen & 2,9 \\
Score médian & 2,2 \\
Plage & 0 à 10 \\
Classification selon le score médian sur l'END, $\mathrm{n}(\%)$ \\
$\leq 2,2$ & $287(50,9)$ \\
$>2,2$ & $277(49,1)$ \\
IDA $^{\mathrm{b}}, \mathrm{n}$ (\%) & \\
Aucune douleur & $114(20,2)$ \\
Douleur légère & $157(27,8)$ \\
Douleur gênante & $194(34,4)$ \\
Douleur pénible & $53(9,4)$ \\
Douleur terrible & $25(4,4)$ \\
Douleur atroce & $21(3,7)$ \\
SCP & \\
Score moyen & 38,0 \\
Score médian & 37,9 \\
Plage & 11,6 à 65,0 \\
SCM & \\
Score moyen & 50,5 \\
Score médian & 52,3 \\
Plage & 20,5 à 69,3 \\
\hline
\end{tabular}

Abréviations : END, échelle numérique de la douleur; IDA, indice de la douleur actuelle; SCM, sommaire de la composante mentale; SCP, sommaire de la composante physique.

a L'END va de 0 (" aucune douleur ») à 10 (" douleur extrême »). Le score médian de l'END a été utilisé pour différencier un groupe souffrant de douleur légère $(\leq 2,2$ / $10)$ et un groupe souffrant de douleur intense $(>2,2 / 10)$.

${ }^{b}$ L'IDA consiste en six combinaisons de chiffres et de mots allant de 0 (" aucune douleur ») à 5 (" douleur atroce»).

c Sommaire de la composante physique du formulaire abrégé à 12 questions de l'Enquête sur la santé (SF-12) ${ }^{31}$ normalisé à une moyenne de 50 , où un score supérieur à 50 est considéré comme supérieur à la moyenne, et un score inférieur à 50 est considéré comme inférieur à la moyenne; les scores s'échelonnent entre 8 et 73.

d Sommaire de la composante mentale du formulaire abrégé à 12 questions de l'Enquête sur la santé (SF-12) ${ }^{31}$ normalisé à une moyenne de 50 , où un score supérieur à 50 est considéré comme supérieur à la moyenne, et un score inférieur à 50 est considéré comme inférieur à la moyenne; les scores s'échelonnent entre 9 et 74.

Le fait de vivre avec d'autres personnes $(p=0,014)$, la présence d'affections concomitantes ( $p=0,031)$ et l'arthrite $(p=0,009)$ ont été associés à une douleur de plus de 2,2/10 dans l'analyse bivariée

TABLEAU 3

Associations bivariées pour l'intensité de la douleur et le modèle final de régression logistique

\begin{tabular}{|c|c|c|c|c|c|}
\hline \multirow[t]{2}{*}{ Variable $^{\mathrm{a}}$} & \multicolumn{3}{|c|}{ Associations bivariées (tests $\chi^{2}$ ) } & \multicolumn{2}{|c|}{ Modèle final de régression ${ }^{b}$} \\
\hline & $\begin{array}{c}0 \text { à } 2,2^{c} \\
\text { n }(\%)\end{array}$ & $\begin{array}{c}2,3 \text { à } 10^{c} \\
\text { n (\%) }\end{array}$ & valeur $p$ & RC (IC à $95 \%)$ & valeur $p$ \\
\hline \multicolumn{6}{|l|}{ Sexe } \\
\hline Femme (0) & $145(48,7)$ & $153(51,3)$ & 0,30 & 1,0 & \multirow{2}{*}{$0,29^{d}$} \\
\hline Homme (1) & $142(53,0)$ & $125(47,0)$ & & $0,8(0,6$ à 1,2$)$ & \\
\hline \multicolumn{6}{|l|}{ Âge } \\
\hline Moins de 65 ans (0) & $104(46,4)$ & $120(53,6)$ & 0,10 & 1,0 & \multirow{2}{*}{$0,052^{d}$} \\
\hline 65 ans et plus (1) & $182(53,5)$ & $158(46,5)$ & & $0,7(0,5$ à 1,0$)$ & \\
\hline \multicolumn{6}{|l|}{ Soutien social } \\
\hline Vivant seul (0) & $117(57,6)$ & $86(42,4)$ & 0,014 & 1,0 & \multirow{5}{*}{0,023} \\
\hline $\begin{array}{l}\text { Vivant avec d'autres per- } \\
\text { sonnes (1) }\end{array}$ & $169(46,8)$ & $192(53,2)$ & & $1,5$ (1,1 à 2,1$)$ & \\
\hline \multicolumn{5}{|l|}{ Tabagisme } & \\
\hline Fumeur & $34(45,3)$ & $41(54,7)$ & \multirow[t]{2}{*}{0,32} & & \\
\hline Non-fumeur & $252(51,5)$ & $237(48,5)$ & & & \\
\hline \multicolumn{6}{|l|}{ Taille de l'ulcère } \\
\hline $5 \mathrm{~cm}^{2}$ et moins & $163(47,9)$ & $177(52,1)$ & \multirow[t]{2}{*}{0,09} & & \\
\hline Plus de $5 \mathrm{~cm}^{2}$ & $123(55,2)$ & $100(44,8)$ & & & \\
\hline \multicolumn{6}{|l|}{ Durée de l'ulcère } \\
\hline 6 mois et moins & $200(48,7)$ & $211(51,3)$ & \multirow[t]{2}{*}{0,11} & & \\
\hline Plus de 6 mois & $86(56,2)$ & $67(43,8)$ & & & \\
\hline \multicolumn{6}{|l|}{ Cause } \\
\hline Veineuse & $251(51,4)$ & $237(48,6)$ & \multirow[t]{2}{*}{0,38} & & \\
\hline Veineuse-mixte & $35(46,1)$ & $41(53,9)$ & & & \\
\hline \multicolumn{6}{|c|}{ Présence d'affections concomitantes } \\
\hline Oui & $163(47,1)$ & $183(52,9)$ & \multirow[t]{2}{*}{0,031} & & \\
\hline Non & $123(56,4)$ & $95(43,6)$ & & & \\
\hline \multicolumn{6}{|c|}{ Maladie cardiovascultaire concomitante } \\
\hline Oui & $61(45,5)$ & $73(54,5)$ & \multirow[t]{2}{*}{0,20} & & \\
\hline Non & $225(52,3)$ & $205(47,7)$ & & & \\
\hline \multicolumn{6}{|l|}{ Arthrite } \\
\hline Oui (1) & $92(32,2)$ & $119(42,8)$ & \multirow[t]{2}{*}{0,009} & $1,6(1,1$ à 2,2$)$ & \multirow{2}{*}{0,016} \\
\hline Non $(0)$ & $194(67,8)$ & $159(57,2)$ & & 1,0 & \\
\hline \multicolumn{6}{|l|}{ Hypertension } \\
\hline Oui & $97(53)$ & $86(47)$ & \multirow[t]{2}{*}{0,45} & & \\
\hline Non & $189(49,6)$ & $192(50,4)$ & & & \\
\hline
\end{tabular}

Abréviations : END, échelle numérique de la douleur; IC, intervalle de confiance; RC, rapport de cotes.

a Les valeurs entre parenthèses représentent le codage des variables utilisées dans le modèle de régression linéaire. Le sexe a été forcé dans le modèle.

${ }^{b}$ Modèle multivarié $r^{2}$ de Cox et Snell $=0,04$.

c L'END va de 0 (« aucune douleur ») à 10 (« douleur extrême »). Le score médian de l'END a été utilisé pour différencier le groupe souffrant de douleur légère $(\leq 2,2 / 10)$ et le groupe souffrant de douleur intense $(>2,2 / 10)$.

d Donnée non statistiquement significative, mais intéressante d'un point de vue empirique (sexe) ou importante sur le plan clinique (âge) et donc conservée dans le modèle.

(tableau 3). Le modèle final de régression logistique a expliqué seulement $4 \%$ de la variation de l'intensité de la douleur. Dans le modèle multivarié, le fait de vivre avec d'autres personnes (RC $=1,5$; IC à $95 \%$ : $1,1$ à $2,1, p=0,023)$ et l'arthrite $(\mathrm{RC}=1,6$; 
IC à $95 \%: 1,1$ à 2,2, $p=0,016)$ étaient associés à la douleur de manière indépendante. Le fait d'être plus âgé $(\mathrm{RC}=0,7$; IC à $95 \%: 0,5$ à $1,0, p=0,052$ ) était également associé à la douleur, mais les données étaient tout juste sous le seuil de la signification statistique. Le fait d'être un homme était associé à une moindre douleur, mais ces données n'étaient pas significatives $(p=0,29)$.

Les tests bivariés pour le SCP (tableau 4) montrent qu'il existe un lien significatif entre une moins bonne QVLS physique et plusieurs facteurs : âge avancé $(p=0,037)$, cause veineuse-mixte ( $p<0,001)$, présence d'affections concomitantes $(p<0,001)$, maladie cardiovasculaire $(p=0,002)$, arthrite ( $p<0,001)$, hypertension $(p=0,027)$ et un score sur l'END supérieur à 2,2 $(p<0,001)$. Le tableau 4 montre également les coefficients et les IC à $95 \%$ correspondants pour le modèle final de régression. Le modèle a expliqué 10,5\% de la variation des résultats. L'arthrite et un score sur l'END supérieur à 2,2 étaient tous deux associés, de manière indépendante, à un score plus faible pour le SCP ( $p<0,001$ dans les deux cas), de même que le fait que l'ulcère soit d'origine mixte ( $p=0,002)$ et qu'il s'agisse d'un ulcère de longue durée $(p=0,006)$. La présence d'une maladie cardiovasculaire concomitante $(p=0,058)$ et un ulcère de plus grande taille ( $p=0,09)$ étaient également associés à un score plus faible pour le SCP. Même si ces données étaient sous le seuil de la signification statistique, elles ont été jugées importantes sur le plan clinique et ont donc été conservées dans le modèle.

En outre, les résultats des tests bivariés et multivariés pour le SCM (tableau 5) révèlent que les personnes de 65 ans et plus obtiennent un meilleur score pour le SCM que celles de moins de 65 ans ( $p=0,006)$, alors que les personnes qui ont déclaré que leur douleur était plus intense ont obtenu un score plus faible pour le SCM $(p<0,001)$. Les données sur le sexe n'étaient pas significatives ( $p=0,61)$. Seule une variable additionnelle, la présence d'une affection concomitante, a montré une tendance vers un score plus faible pour le SCM $(p=0,058)$. Le tableau 5 montre également les coeffi-
TABLEAU 4

Associations bivariées pour le sommaire de la composante physique du formulaire SF-12 et le modèle final de régression linéaire multivarié

\begin{tabular}{|c|c|c|c|c|}
\hline \multirow[t]{2}{*}{ Variable $^{\mathrm{a}}$} & \multicolumn{2}{|c|}{ Associations bivariées ${ }^{\mathbf{b}}$} & \multicolumn{2}{|c|}{ Modèle final de régression } \\
\hline & Moyenne (É.-T.) & valeur $p$ & Coefficient (IC à $95 \%$ ) & valeur $p$ \\
\hline Constante du modèle & & & $42,8(41,0$ à 44,6$)$ & \\
\hline \multicolumn{5}{|l|}{ Sexe } \\
\hline Femme (0) & $37,3(9,7)$ & 0,077 & $1,1(-0,5$ à 2,8$)$ & 0,18 \\
\hline Homme (1) & $38,9(10,7)$ & & & \\
\hline \multicolumn{5}{|l|}{ Âge } \\
\hline Moins de 65 ans & $39,1(9,9)$ & 0,037 & & \\
\hline 65 ans et plus & $37,3(10,4)$ & & & \\
\hline \multicolumn{5}{|l|}{ Tabagisme } \\
\hline Fumeur actuel & $39,3(10,3)$ & 0,25 & & \\
\hline Non-fumeur & $37,5(10,2)$ & & & \\
\hline \multicolumn{5}{|l|}{ Soutien social } \\
\hline Vivant seul & $37,5(10,2)$ & 0,34 & & \\
\hline Vivant avec d'autres personnes & $38,4(10,2)$ & & & \\
\hline \multicolumn{5}{|l|}{ Taille de l'ulcère } \\
\hline $5 \mathrm{~cm}^{2}$ et moins $(0)$ & $38,6(10,8)$ & 0,10 & $-1,4(-3,1$ à 0,2$)$ & $0,09^{c}$ \\
\hline Plus de $5 \mathrm{~cm}^{2}(1)$ & $37,2(9,1)$ & & & \\
\hline \multicolumn{5}{|l|}{ Durée de l'ulcère } \\
\hline 6 mois et moins $(0)$ & $38,5(11,1)$ & 0,09 & $-2,6(-4,5$ à $-0,7)$ & 0,006 \\
\hline Plus de 6 mois (1) & $36,9(9,3)$ & & & \\
\hline \multicolumn{5}{|l|}{ Cause de l'ulcère à la jambe } \\
\hline Veineuse (0) & $38,7(10,2)$ & $<0,001$ & $-3,9(-6,4$ à $-1,5)$ & 0,002 \\
\hline Veineuse-mixte (1) & $33,6(9,2)$ & & & \\
\hline \multicolumn{5}{|l|}{ Présence d'affections concomitantes } \\
\hline Oui & $36,5(10,5)$ & $<0,001$ & & \\
\hline Non & $40,5(9,2)$ & & & \\
\hline \multicolumn{5}{|l|}{ Maladie cardiovasculaire } \\
\hline Oui (1) & $35,4(11,0)$ & 0,002 & $-2,0(-3,9$ à 0,1$)$ & $0,058^{\mathrm{C}}$ \\
\hline Non $(0)$ & $38,9(9,7)$ & & & \\
\hline \multicolumn{5}{|l|}{ Arthrite } \\
\hline Oui (1) & $35,3(10,5)$ & $<0,001$ & $-3,4(-5,1$ à $-1,6)$ & $<0,001$ \\
\hline Non $(0)$ & $39,7(9,7)$ & & & \\
\hline \multicolumn{5}{|l|}{ Hypertension } \\
\hline Oui & $36,7(9,8)$ & 0,027 & & \\
\hline Non & $38,7(10,3)$ & & & \\
\hline \multicolumn{5}{|l|}{ Douleur $^{d}$} \\
\hline$\leq 2,2(0)$ & $39,9(9,7)$ & $<0,001$ & $-3,5(-5,1$ à $-1,9)$ & $<0,001$ \\
\hline$>2,2(1)$ & $36,1(10,2)$ & & & \\
\hline
\end{tabular}

Abréviations : END, échelle numérique de la douleur; IC, intervalle de confiance; $R^{2}$, coefficient de détermination; É.-T., écart-type; SF-12, formulaire abrégé comportant 12 questions de l'Enquête sur la santé.

a Les valeurs entre parenthèses représentent le codage des variables utilisées dans le modèle de régression linéaire. Le sexe a été forcé dans le modèle. $R^{2}$ ajusté pour le modèle $=0,11$.

${ }^{\mathrm{b}}$ Résultats des tests $t$ de Student.

' Donnée non statistiquement significative, mais intéressante d'un point de vue empirique (sexe) ou importante sur le plan clinique (taille, maladie cardiovasculaire) et donc conservée dans le modèle.

d D'après le score médian sur l'END utilisé pour différencier le groupe souffrant de douleur légère $(\leq 2,2 / 10)$ et le groupe souffrant de douleur intense $(>2,2 / 10)$. 
TABLEAU 5

Associations bivariées (tests $t$ de Student) pour le sommaire de la composante mentale du formulaire SF-12 et le modèle final de régression linéaire multivarié

\begin{tabular}{|c|c|c|c|c|}
\hline \multirow[t]{2}{*}{ Variable $^{\mathrm{a}}$} & \multicolumn{2}{|c|}{ Associations bivariées ${ }^{b}$} & \multicolumn{2}{|c|}{ Modèle final de régression } \\
\hline & Moyenne (É.-T.) & valeur $p$ & Coefficient (IC à $95 \%$ ) & valeur $f$ \\
\hline Constante du modèle & & & $53,2(54,1$ à 55,5$)$ & \\
\hline \multicolumn{5}{|l|}{ Sexe } \\
\hline Femme (0) & $50,3(10,6)$ & 0,61 & $0,4(-1,3$ à 2,1$)$ & 0,63 \\
\hline Homme (1) & $50,8(10,2)$ & & & \\
\hline \multicolumn{5}{|l|}{ Âge } \\
\hline Moins de 65 ans (0) & $49,0(11,0)$ & 0,006 & $2,6(0,8$ à 4,4$)$ & 0,005 \\
\hline 65 ans et plus (1) & $51,5(9,8)$ & & & \\
\hline \multicolumn{5}{|l|}{ Tabagisme } \\
\hline Fumeur & $48,8(11,1)$ & 0,12 & & \\
\hline Non-fumeur & $50,8(10,3)$ & & & \\
\hline \multicolumn{5}{|l|}{ Soutien social } \\
\hline Vivant seul & $50,0(10,6)$ & 0,46 & & \\
\hline Vivant avec d'autres personnes & $50,3(10,3)$ & & & \\
\hline \multicolumn{5}{|l|}{ Taille de l’ulcère } \\
\hline $5 \mathrm{~cm}^{2}$ et moins & $50,5(10,4)$ & 0,83 & & \\
\hline Plus de $5 \mathrm{~cm}^{2}$ & $50,7(10,4)$ & & & \\
\hline \multicolumn{5}{|l|}{ Durée de l'ulcère } \\
\hline 6 mois et moins $(0)$ & $50,9(10,1)$ & 0,16 & $-1,9(-3,9$ à 0,0$)$ & 0,053 \\
\hline Plus de 6 mois (1) & $49,5(10,6)$ & & & \\
\hline \multicolumn{5}{|l|}{ Cause de l'ulcère à la jambe } \\
\hline Veineuse & $50,6(10,5)$ & 0,59 & & \\
\hline Veineuse-mixte & $50,0(10,0)$ & & & \\
\hline \multicolumn{5}{|l|}{ Présence d'affections concomitantes } \\
\hline Oui (1) & $50,0(10,8)$ & 0,058 & $-2,3(-4,2$ à $-0,5)$ & 0,014 \\
\hline Non $(0)$ & $51,4(9,7)$ & & & \\
\hline \multicolumn{5}{|l|}{ Maladie cardiovasculaire } \\
\hline Oui & $50,1(10,6)$ & 0,78 & & \\
\hline Non & $50,7(10,3)$ & & & \\
\hline \multicolumn{5}{|l|}{ Arthrite } \\
\hline Oui & $50,3(10,8)$ & 0,26 & & \\
\hline Non & $50,7(10,1)$ & & & \\
\hline \multicolumn{5}{|l|}{ Hypertension } \\
\hline Oui & $51,0(10,2)$ & 0,56 & & \\
\hline Non & $50,3(10,4)$ & & & \\
\hline \multicolumn{5}{|l|}{ Douleur $^{d}$} \\
\hline$\leq 2,2(0)$ & $53,1(9,5)$ & $<0,001$ & $-4,9(-5,6$ à $-3,2)$ & $<0,001$ \\
\hline$>2,2(1)$ & $47,9(10,6)$ & & & \\
\hline
\end{tabular}

Abréviations : END, échelle numérique de la douleur; É.-T., écart-type; IC, intervalle de confiance; $R^{2}$, coefficient de détermination; SF-12, formulaire abrégé à 12 questions de l'Enquête sur la santé.

${ }^{a}$ Les valeurs entre parenthèses représentent le codage des variables utilisées dans le modèle de régression linéaire. Le sexe a été forcé dans le modèle. $R^{2}$ ajusté pour le modèle $=0,08$.

b Résultats des tests $t$ de Student.

c Donnée non statistiquement significative, mais intéressante d'un point de vue empirique (sexe) ou importante sur le plan clinique (durée) et donc conservée dans le modèle.

d D'après le score médian sur l'END utilisé pour différencier le groupe souffrant de douleur légère $(\leq 2,2 / 10)$ et le groupe souffrant de douleur intense $(>2,2 / 10)$. cients et les IC à $95 \%$ correspondants du modèle final de régression. Le modèle a expliqué $7,8 \%$ de la variation des résultats. L’âge avancé a été associé à un meilleur score pour le SCM ( $p=0,005$ ), tandis que la présence d'une affection concomitante et une douleur plus intense ont toutes deux été associées à un score plus faible pour le $\operatorname{SCM}(p=0,014$ et $p<0,001$, respectivement). Le fait de présenter un ulcère de longue durée ( $p=0,053)$ a également été associé à un score plus faible pour le SCM; même si ces données étaient sous le seuil de la signification statistique, elles ont été jugées importantes sur le plan clinique et ont été conservées dans le modèle.

\section{Analyse}

À notre connaissance, il s'agit de l'une des plus importantes études visant à examiner les liens entre des variables cliniques mesurées de façon systématique et la QVLS chez une population validée cliniquement de patients souffrant d'ulcères veineux. Nous avons obtenu des données substantielles pour confirmer les liens entre les variables individuelles, environnementales et cliniques, la douleur et la QVLS dans cette population.

En général, les femmes ont fait état d'une douleur quelque peu plus intense et ont obtenu des scores plus faibles pour le SCP et le SCM, mais les résultats étaient non significatifs sur le plan statistique dans chacun des trois modèles. Les participants plus jeunes ont fait état d'une douleur plus importante et ont obtenu un score plus faible pour le SCM, mais les données relatives à l'âge étaient tout juste sous le seuil de la signification statistique dans le modèle final de régression du SCP. Comme le SCP diminue avec l'âge ${ }^{40}$, nous avions anticipé que le groupe des participants plus âgés allait obtenir un score relativement faible pour le SCP. La grande taille de l'échantillon assurait une bonne représentation des jeunes adultes et des adultes plus âgés, et l'absence d'effet n'est donc pas due à l'homogénéité de la variable indépendante. Cela peut plutôt s'expliquer par le fait que le groupe des participants jeunes a obtenu des scores plus faibles que prévu pour le SCP, ce qui a annulé l'effet de l'âge. Cela pourrait 
venir appuyer les résultats d'études antérieures qui indiquaient que l'écart enregistré par rapport aux normes relatives à la QVLS était plus important chez les personnes de moins de 65 ans que chez les personnes plus âgées ${ }^{28}$, ce que confirme le fait que les deux groupes ont obtenu pour le SCP un score moyen nettement inférieur au score enregistré chez la population de référence au Canada ${ }^{40}$. Il est également possible qu'il y ait eu une colinéarité avec certaines des autres variables, par exemple l'arthrite, une affection beaucoup plus courante chez le groupe plus âgé. Cependant, le fait de retirer certaines variables individuelles et de réexécuter les modèles a eu peu d'effet sur les autres coefficients de régression, ce qui laisse croire que la colinéarité était minime.

Un lien a été établi entre le fait d'être jeune et l'obtention d'un score plus élevé touchant la douleur, malgré l'augmentation probable de l'incidence des affections concomitantes associées à la douleur chez le groupe plus âgé. En ce qui concerne les caractéristiques des ulcères, aucune différence significative sur le plan statistique n’a été observée entre les jeunes adultes et les adultes plus âgés. Il est par conséquent improbable qu'un fondement biologique explique la différence quant à l'intensité de la douleur. Il est possible que les jeunes adultes aient des attentes plus élevées et qu'ils soient généralement plus actifs que les adultes plus âgés en raison de leur travail et de leur famille, ce qui pourrait exacerber la douleur. Malheureusement, nous ne pouvons pas vérifier cette hypothèse, car notre étude ne comprenait aucune mesure de l'état fonctionnel. Il se pourrait aussi que les jeunes adultes et les adultes plus âgés ressentent ou déclarent différemment l'intensité de la douleur. Toutefois, il n'existe aucune preuve pour appuyer cette affirmation, et la majorité des publications suggèrent que l'intensité de la douleur augmente avec l'âge ${ }^{42}$. Comme prévu, nous avons observé un lien très significatif et négatif entre le SCP et le SCM et la douleur plus intense.

Ni la taille ni la durée des ulcères n’ont pu être associées de façon significative à l'intensité de la douleur dans les tests bivariés et multivariés, même si les participants qui présentaient des ulcères de petite taille et de courte durée ont généralement fait état d'une douleur plus intense. Le lien entre la taille ou la durée des ulcères et l'intensité de la douleur n'ont fait l'objet que d'un nombre limité d'études. Pieper et collab. ${ }^{43}$ ont établi une corrélation modérée entre les très grandes plaies et l'intensité de la douleur ( $r$ de Pearson $=0,44)$. Dans le cadre de leur vaste étude transversale, Walters et collab. ${ }^{16}$ ont observé un lien entre les ulcères veineux de plus de $5,6 \mathrm{~cm}^{2}$ et un score élevé en ce qui concerne la douleur ressentie le jour. Nos résultats ne confirment ceux d'aucune de ces études, peutêtre en raison de différences d'ordre méthodologique. L'étude menée par Pieper et collab. ${ }^{43}$ portait sur un petit nombre de jeunes adultes qui présentaient d'importants ulcères veineux et qui avaient des problèmes de toxicomanie. De plus, des quatre mesures temporelles de la douleur, seule l'intensité de la douleur ressentie le jour était associée à la taille de l'ulcère. Il se peut que le lien entre l'intensité de la douleur et les caractéristiques des ulcères ne soit ni simple ni linéaire. Sur le plan clinique, les petits ulcères associés à une incontinence de veine perforante ou à des taches hypopigmentées causées par l'atrophie blanche pourraient être associés à une douleur intense $\mathrm{e}^{44}$, et les ulcères présents depuis longtemps pourraient être associés à une douleur moins intense $\mathrm{2}^{24,45}$. Le lien entre les caractéristiques des ulcères, la gravité de la maladie et la douleur mérite d'être étudié de façon plus approfondie.

Les modèles finaux de régression n’ont expliqué qu'une petite proportion de la variation de l'intensité de la douleur et de la QVLS. Cela peut être attribuable à plusieurs facteurs. L'un d'eux pourrait être la faiblesse des associations causales dans la démarche du modèle de Wilson et Cleary $^{15}$, mais il est plus probable que les mesures relatives aux caractéristiques biologiques et à l'état des symptômes soient incomplètes et qu'elles représentent un sous-ensemble d'un ensemble beaucoup plus grand. La meilleure façon de conceptualiser les caractéristiques biologiques n'est pas claire ${ }^{46}$. La taille et la durée sont des indicateurs de la gravité des ulcères bien acceptés cliniquement, mais il se peut que la liste à partir de laquelle les patients ont déclaré leurs affections concomitantes, initialement conçue pour faciliter le processus d'établissement d'un diagnostic différentiel, ait été un indicateur faible de la gravité de l'affection concomitante, une mesure potentiellement importante dans cette population principalement plus âgée. De plus, nous avons choisi comme indicateur de la douleur son intensité, seul indicateur parmi les divers symptômes associés aux ulcères. En outre, il se pourrait que l'END n'ait pas permis de saisir certains aspects pertinents de l'expérience de la douleur, par exemple la composante affective de la douleur et les interférences liées à la douleur. Troisièmement, il est possible qu'un certain nombre de variables qui contribuent à la QVLS, par exemple l'état fonctionnel, n'aient pas été intégrées à ces deux essais. Chose certaine, il y a suffisamment d'éléments prouvant que l'ulcération active altère la fonction physique. L'inclusion d'une mesure solide de l'état fonctionnel aurait seulement acccru la puissance explicative des modèles.

Même si les modèles n'ont fourni qu'une explication limitée de la variation, nous avons observé des liens importants sur le plan clinique entre les caractéristiques sociodémographiques et biologiques, l'intensité de la douleur et la QVLS. Voici un scénario clinique auquel le personnel infirmier est souvent confronté et qui illustre très bien ces liens : prenons l'exemple d'une personne de 67 ans souffrant d'un ulcère veineux depuis 8 mois. L'arthrose complique l'expérience de la douleur du patient, qui évalue sa douleur à 4 sur une échelle de 0 à 10 . D’après ces caractéristiques et les coefficients du modèle de régression, nous avons calculé que la QVLS physique du patient a diminué de 14,7 points et que sa QVLS mentale a diminué de 7,6 points. Cette diminution du score concernant la QVLS est importante sur le plan clinique et elle illustre les liens importants qui existent entre les caractéristiques cliniques mesurées de façon systématique et la QVLS.

\section{Points forts et limites}

Notre étude comportait plusieurs limites. Premièrement, la causalité ne peut pas 
être étudiée au moyen d'une méthode transversale. Deuxièmement, comme il s'agissait d'une analyse secondaire, les variables de l'étude étaient conçues pour répondre à d'autres questions. Par exemple, il aurait peut-être été plus efficace de mesurer l'état des symptômes à l'aide d'un outil éprouvé portant sur une maladie précise et axé sur les symptômes, en plus de l'intensité de la douleur. D'autres concepts importants tels que l'état fonctionnel n'étaient pas inclus dans l'étude.

Parmi les forces de notre étude figurent la taille importante de l'échantillon et l'utilisation d'outils éprouvés pour mesurer la douleur et la QVLS. De plus, les principaux ECR sur lesquels repose cette étude utilisaient des méthodes cohérentes et éprouvées pour évaluer la taille et la durée des ulcères. Enfin, il est probable que l'important échantillon de sujets provenant de milieux urbains et ruraux soit raisonnablement représentatif des populations cliniques qui reçoivent des traitements pour des ulcères veineux.

Les travaux de recherche futurs devraient viser à définir ou tester des méthodes de mesure de l'état des symptômes portant sur des maladies précises, ce qui constituerait la prochaine étape de l'élaboration d'un modèle de la QVLS pertinent sur le plan clinique. De plus, les concepts clés tels que les affections concomitantes et la gravité de la maladie doivent être conceptualisés davantage. Enfin, les études à venir devraient tenir compte de l'âge, une variable importante, et inclure une mesure solide de l'état fonctionnel. En effet, l'altération de la fonction physique et d'autres aspects de l'état fonctionnel en lien avec les attentes propres à l'âge pourraient jouer un rôle clé dans la médiation des effets de l'âge et de la douleur sur la QVLS.

L'évaluation de la QVLS est un processus complexe. Il est probable que les caractéristiques des ulcères et l'intensité de la douleur, deux variables fréquemment mesurées par les cliniciens, ne représentent qu'un aspect limité de la QVLS. À elles seules, ces deux variables ne constituent pas des paramètres suffisants pour évaluer la QVLS. L'incidence de l'ulcération sur la vie quotidienne est un sujet qu'il est important d'aborder avec les patients dès le départ, et qui devrait être pris en compte lors des évaluations et de la planification des soins. Comprendre l'incidence des symptômes sur l'état fonctionnel pourrait se révéler crucial au moment d'établir des objectifs prioritaires et d'évaluer les interventions du point de vue du patient.

\section{Remerciements}

L'essai contrôlé randomisé Nurse Clinic Versus Home Delivery of Evidence-based Community Leg Ulcer Care a été financé par une subvention des Instituts de recherche en santé du Canada (MOP n ${ }^{\circ}$ 42497), qui remplacent le Conseil de recherches médicales. L'essai Canadian Bandaging a été financé par une subvention des Instituts de recherche en santé du Canada (IRSC $n^{\circ}$ 63175, numéro standard international pour les essais contrôlés randomisés ISRCTN37782978).

\section{Références}

1. Callam MJ, Harper DR, Dale JJ, Ruckley CV. Chronic ulcer of the leg: clinical history. $\mathrm{Br}$ Med J. 1987;294:1389-91.

2. Graham ID, Harrison MB, Nelson EA, Lorimer K, Fisher A. Prevalence of lowerlimb ulceration: a systematic review of prevalence studies. Adv Skin Wound Care. 2003;16(6):305-16.

3. Callam MJ. Leg ulcer and chronic venous insufficiency in the community. In: Ruckley CV, Fowkes FG, Bradbury A, editors. Venous disease: epidemiology, management and delivery of care. London: Springer; 1999. 15-25.

4. Harrison MB, Graham ID, Lorimer K, Friedberg E, Pierscianowski T, Brandys T. Leg-ulcer care in the community, before and after implementation of an evidence-based service. CMAJ. 2005;172(11):1447-52.

5. Harrison MB, Graham ID, Lorimer $\mathrm{K}$ et collab. Nurse clinic versus home delivery of evidence-based community leg ulcer care: a randomized health services trial. BMC Health Serv Res. 2008;8(1):243.
6. Nelson EA. Health related quality of life measurement. EWMA Journal. 2002;2(1):5-7.

7. Barwell JR, Davies CE, Deacon F et collab. Comparison of surgery and compression with compression alone in chronic venous ulceration (ESCHAR study): randomised controlled trial. Lancet. 2004;363(9424):1854-9.

8. Morrell CJ, Walters SJ, Dixon S, Collins KA, Brereton LML, Peters J. Cost effectiveness of community leg ulcer clinics: randomised controlled trial. BMJ. 1999;316(7143):1487-91.

9. Persoon A, Heinen MM, van der Vleuten CJ, de Rooij MJ, van de Kerkhof PC, van Achterburg T. Leg ulcers: a review of their impact on daily life. J Clin Nurs. 2004; 13(3):341-54.

10. Herber OR, Schnepp W, Rieger MA. A systematic review on the impact of leg ulceration on patients' quality of life. Health Qual Life Outcomes. 2007;5:44-66.

11. Briggs M, Flemming K. Living with leg ulceration: a synthesis of qualitative research. J Adv Nurs. 2007;59(4):319-28.

12. Green J, Jester R. Health-related quality of life and chronic venous leg ulceration: part 2. Br J Community Nurs. 2010;15(3):S4-6, S8, S10, passim.

13. Hareendran A, Bradbury A, Budd J et collab. Measuring the impact of venous leg ulcers on quality of life. J Wound Care. 2005;14(2):53-7.

14. Gonzalez-Consuegra RV, Verdu J. Quality of life in people with venous leg ulcers: an integrative review. J Adv Nurs. 2011;67(5): 926-44.

15. Wilson IB, Cleary PD. Linking clinical variables with health-related quality of life. A conceptual model of patient outcomes. JAMA. 1995;273(1):59-65.

16. Walters SJ, Morrell CJ, Dixon S. Measuring health-related quality of life in patients with venous leg ulcers. Qual Life Res. 1999; 8:327-36. 
17. Iglesias CP, Birks Y, Nelson EA, Scanlon E, Cullum NA. Quality of life of people with venous leg ulcers: a comparison of the discriminative and responsive characteristics of two generic and a disease specific instrument. Qual Life Res. 2005;14:1705-18.

18. Franks PJ, Moffatt CJ. Do clinical and social factors predict quality of life in leg ulceration? Int J Low Extrem Wounds. 2006;5(4): 236-43.

19. Nemeth KA, Harrison MB, Graham ID, Burke S. Pain in pure and mixed aetiology venous leg ulcers: a three-phase point prevalence study. J Wound Care. 2003; 12(9):336-40.

20. Moffatt CJ, Harper P. Leg ulcers (access to clinical education). New York (NY): Churchill Livingstone; 1997.

21. Phillips T, Stanton B, Provan A, Lew R. A study of the impact of leg ulcers on quality of life: financial, social, and psychologic implications. J Am Acad Dermatol. 1994:31:49-53.

22. Krasner D. Painful venous ulcers: themes and stories about living with the pain and suffering. J Wound Ostomy Continence Nurs. 1998;25:158-68.

23. Franks PJ, Moffatt CJ. Quality of life issues in chronic wound management. $\mathrm{Br} \mathrm{J}$ Community Nurs. 1999;4(6):283-9.

24. Price P, Harding K. Measuring healthrelated quality of life in patients with chronic leg ulcers. Wounds. 1996;8(3):91-4.

25. Franks PJ, McCullagh L, Moffatt CJ. Assessing quality of life in patients with chronic leg ulceration using the Medical Outcomes Short Form-36 Questionnaire. Ostomy Wound Manage. 2003;49(2):26-37.

26. Jull A, Walker N, Hackett M et collab. Leg ulceration and perceived health: a population based case-control study. Age Ageing. 2004;33:236-41.

27. Lindholm C, Bjellerup M, Christensen OB, Zederfeldt B. Quality of life in chronic leg ulcer patients. An assessment according to the Nottingham Health Profile. Acta Derm Venereol. 1993;73(6):440-443.

28. Franks PJ, Moffatt CJ. Who suffers most from leg ulceration? J Wound Care. 1998; $7(8): 383-5$.
29. Moffatt CJ, Franks PJ, Doherty DC, Smithdale R, Martin R. Sociodemographic factors in chronic leg ulceration. $\mathrm{Br} \mathrm{J}$ Dermatol. 2006;155:307-12.

30. Harrison MB, Vandenkerkhof EG, Hopman WM, Graham ID, Carley ME, Nelson EA. The Canadian Bandaging Trial: evidenceinformed leg ulcer care and the effectiveness of two compression technologies. BMC Nurs. 2011;10(1):20.

31. Ware JE, Kosinski M, Keller SD. SF-36 Physical and mental health summary scales: a user's manual and interpretation guide. Boston (MA): The Health Institute, New England Medical Center; 1994.

32. Ware JE, Sherbourne CD. The MOS 36-item short-form health survey (SF-36). I: conceptual framework and time selection. Med Care. 1992;30:473-83.

33. Ware JE. SF-36 Health Survey Update. In: Maruish ME, , editor. The use of psychological testing for planning and outcomes assessment 3rd ed. Mahwah (NJ): Lawrence Erlbaum Associates; 2004. 693-718.

34. Charles H. Does leg ulcer treatment improve patients' quality of life? J Wound Care. 2004;13(6):209-13.

35. Harrison MB, VanDenKerkhof E, Hopman WM, Graham ID, Lorimer K, Carley M. Evidence-informed leg ulcer care: a cohort study comparing outcomes of individuals choosing nurse-led clinic or home care. Ostomy Wound Manage. 2011;57(8):38-45.

36. Melzack R. The short-form McGill Pain Questionnaire. Pain. 1987;30(2):191-7.

37. Briggs M, Bennett MI, Closs SJ, Cocks K. Painful leg ulceration: a prospective, longitudinal cohort study. Wound Repair Regen. 2007;15:186-91.

38. Charles H. Venous leg ulcer pain and its characteristics. J Tissue Viability. 2002;12(4):154-8.

39. Noonan L, Burge SM. Venous leg ulcers: Is pain a problem? Phlebology. 1998;13:14-9.
40. Association des infirmières et infirmiers autorisés de l'Ontario (RNAO). Learning package. Assessment and management of venous leg ulcers [Internet]. Nursing Best Practice Guidelines Program. Toronto (Ont.): Association des infirmières et infirmiers autorisés de l'Ontario; 2006 [consultation le 2 mai 2012]. PDF (1,14 Mo) téléchargeable à partir du lien : http://rnao.ca/sites/rnao-ca /files/Assessment_and_Management_of _Venous_Leg_Ulcers_-_Learning_Package.pdf

41. Hopman WM, Towheed T, Anastassiades T et collab. Canadian normative data for the SF-36 health survey. Canadian Multicentre Osteoporosis Study Research Group. CMAJ. 2000;163(3):265-71.

42. Reitsma ML, Tranmer JE, Buchanan DM, Vandenkerkhof EG. Prévalence de la douleur chronique et des limitations fonctionnelles qui lui sont associées au Canada entre 1994 et 2008. Maladies chroniques et blessures au Canada. 2011;31(4):157-64 .

43. Pieper B, Rossi R, Templin T. Pain associated with venous ulcers in injecting drug users. Ostomy Wound Manage. 1998; 44(11):54-67.

44. Kunimoto BT. Assessment of venous leg ulcers: an in-depth discussion of a literature-guided approach. Ostomy Wound Manage. 2001;47(5):38-53.

45. Husband LL. Venous ulceration: the pattern of pain and the paradox. Clin Eff Nurs. 2001;5:35-40.

46. Sousa KH, Holzemer WL, Henry SB, Slaughter R. Dimensions of health-related quality of life in persons living with HIV disease. J Adv Nurs. 1999;29(1):178-87. 